\title{
To and fro motion for the hydrogen atom in a circularly polarized microwave field
}

\author{
Mercè Olléa \\ ${ }^{a}$ Universitat Politècnica de Catalunya, Escola Tècnica Superior d'Enginyeria Industrial de \\ Barcelona, Av Diagona 647, 08028 Barcelona, Spain
}

\begin{abstract}
We study the problem of the hydrogen atom interacting with a circularly polarized microwave field, and more specifically, we focus on the so called to and fro motion, that is, the erratic trajectories described by the electron making several large distance excursions and close passages to the nucleus. The skeleton of such trajectories is based on the so called ejection-collision orbits (ECO), that is, orbits ejected from the nucleus, describing several far/close passages to the origin and finally colliding with it. The computation and continuation of families of ECO, as well as their bifurcations is analysed, and finally the consequences of such orbits to explain to and from motion as well as ionization of an electron by the external field are also described.
\end{abstract}

Keywords: regularization, ejection-collision orbits, invariant manifolds, global dynamics

2010 MSC: 70F07, 70F15

\section{Introduction}

The study of the $n$-body problem, and in particular, the 3-body problem in Celestial Mechanics has been the keystone to advance in the understanding of different aspects of classical mechanics -both from a physical and mathematical 5 point of view-. Chaotic dynamics is an intriguing one. However, chaos appears

\footnotetext{
${ }^{*}$ Corresponding author

Email address: Merce.0lle@upc.edu (Mercè Ollé)
}

Preprint submitted to Communications in Nonlinear Science and Numerical SimulationMay 17, 2017 
also in the microscopic or quantum problems. It is remarkable how the study of the Restricted three body problem - which may be regarded as a perturbation of the Kepler problem-, is a paradigm problem in the macroscale mechanics and a vast literature has been, and still is, devoted to it (see [3], [5], [6] and [16] and references therein). In the microscale or quantum mechanics, there exists an analogue problem which is the hydrogen atom interaction with a circularly polarized electromagnetic field, called from now on the CP problem. This problem, in rotating coordinates becomes a Keplerian problem plus a perturbation depending on one parameter and the equations of motion for the electron can be written as a Hamiltonian system of ordinary differential equations (ODE) which has a unique singularity that corresponds to the collision with the nucleus (located at the origin). Thus, the tools from a dynamical system approach that are applied for the RTBP may be used in the CP problem.

Many references have been devoted to this problem (see for example [1], [2],

$20 \quad[4]$ and [12] and references therein); the last one being the first systematic study of the relevant invariant objects in phase space which control the dynamics, but more focused on ionizing/escaping orbits. However none of them analyse a mechanism that explains the naive behaviour of the so called to and fro motion, that is, the behaviour of the electron when it describes a trajectory with several 25 far and close passages to the nucleus in an erratic or chaotic way. An approach to this mechanism is studied in [1]; nevertheless, in that paper, the authors considered different sets of initial conditions and integrated numerically the CP system of ODE but stopped the numerical integration when the electron was close to the nucleus due to the presence of the singularity. Therefore no results about the behavior of trajectories passing very close of the origin were given. In the present paper, we regularize the equations of motion to remove the singularity, so this difficulty is completely overcome. More precisely, we study orbits that eject from (or collide with) the origin and have several close passages to it. The skeleton of the to and fro motion consists of what we call $n$ ejectioncollision orbits (denoted from now on as $n$-ECO). An $n$-ECO is a trajectory that ejects from the nucleus (origin), has $2 n-1$ extrema in the distance to the origin 
(typically corresponding to $n$ maxima and $n-1$ minima), and collides with the nucleus. We will show how these ECO are simply heteroclinic connections between different equilibrium points and we study their existence when varying

40 the energy. This approach was originally applied in the RTBP, and partial analytical results are obtained in [8], [9] and [10] for the circular RTBP and in [11], [15] for the elliptic case. A massive numerical analysis for the circular RTBP is recently carried out in [14]. Finally we will discuss the role that such ECO play on the global dynamics of the CP problem, concerning not only the to and fro motion, but the non-return (or ionization) as well.

The paper is organized as follows: in Section 2 we provide the system of ordinary differential equations (ODE) governing the motion for the electron in a rotating system of coordinates. Some basic and known properties of this problem, concerning equilibrium points, periodic orbits and possible regions of motion, are shortly recalled. In Section 3 we explain the methodology to deal with ECO: the regularization of the singularity at the origin of the system of ODE is carried out, the collision manifold is studied (which provides an insight about how orbits passing close to the collision will behave) and the strategies to compute ECO and the organization of ECO in families (and bifurcations) are described. The convenience to use suitable Poincaré sections is also discussed. Section 4 is devoted to provide and describe the results obtained from massive simulations. The possibility of having ejection orbits that finally ionize is also explained taking into account the values of the energy of the CP problem. Fynally, some conclusions are drawn in Section 5.

We finally remark that the numerical integrations of the systems of ODE done along the paper use a Taylor method implemented on a robust, fast and accurate software package by Jorba and Zou ([7]).

\section{Description of the CP problem. Main features}

In order to have a self contained paper, we present the main features of this 65 problem. The details can be found in [1]. 
We consider the relative motion of a hydrogen atom submitted to a circularly polarized (CP) microwave, where the pulse of the microwave field is taken with a flat-top shape, that is, the field amplitude is ramped up in time until it achieves a final, constant amplitude. In this study we ignore the ramping and just consider the dynamics after the flat-top has been reached (see [2] for a discussion of the consequences of the initial ramp).

The Hamiltonian for the electron of the hydrogen atom (in the limit of an infinitely massive nucleus and in atomic units $m_{e}=\bar{h}=e=1$ ) subjected to a $\mathrm{CP}$ microwave field is the following:

$$
\tilde{H}\left(X, Y, X^{\prime}, Y^{\prime}\right)=\frac{1}{2}\left(X^{\prime 2}+Y^{\prime 2}+Z^{\prime 2}\right)-\frac{1}{R}+F(X \cos \omega s+Y \sin \omega s),
$$

where $(X, Y, Z)$ are the position coordinates, $R^{2}=X^{2}+Y^{2}+Z^{2}, s$ is the time, $'=\frac{d}{d s}, \omega$ is the angular frequency of the microwave field and $F>0$ is the field strength (see [2]). We will consider the motion in the planar case, that is for $Z=0$. Furthermore, we take a rotating frame with the CP field, that is, $(x, y)$ coordinates such that

$$
\left(\begin{array}{l}
X \\
Y
\end{array}\right)=\left(\begin{array}{cc}
\cos \omega s & -\sin \omega s \\
\sin \omega s & \cos \omega s
\end{array}\right)\left(\begin{array}{l}
x \\
y
\end{array}\right)
$$

Defining now the momenta $p_{x}=\dot{x}-\omega y$ and $p_{y}=\dot{y}+\omega x$, the transformed Hamiltonian becomes

$$
\hat{H}\left(x, y, p_{x}, p_{y}\right)=\frac{1}{2}\left(p_{x}^{2}+p_{y}^{2}\right)-\omega\left(x p_{y}-y p_{x}\right)-\frac{1}{R}+F x,
$$

being $R=\sqrt{x^{2}+y^{2}}$.

Now we simplify this Hamiltonian re-scaling time and distances. We define a new time $t=\omega s$ and consider the symplectic change of coordinates with 75 multiplier $\omega^{-1 / 3}$, more precisely,

$$
(x, y)=\omega^{-2 / 3}(\bar{x}, \bar{y}), \quad\left(p_{x}, p_{y}\right)=\omega^{1 / 3}\left(\bar{p}_{x}, \bar{p}_{y}\right) .
$$

The transformed Hamiltonian becomes in the new variables (for simplicity, we drop the bar and we keep the same names for the position and momentum 
coordinates),

$$
H\left(x, y, p_{x}, p_{y}\right)=\frac{1}{2}\left(p_{x}^{2}+p_{y}^{2}\right)-x p_{y}+y p_{x}-\frac{1}{r}+K x,
$$

where $K=F / w^{4 / 3}>0$ and now $r=\sqrt{x^{2}+y^{2}}, p_{x}=\frac{d x}{d t}-y$ and $p_{y}=\frac{d y}{d t}+x$.

A first remark is that we obtain an autonomous Hamiltonian, depending on the parameter $K>0$, with two degrees of freedom, which turns out to be a perturbation of the well known Kepler problem. So for $K$ very small, we should expect the typical dynamics of a Hamiltonian system close to an integrable one, but of course with the features of a non integrable one. We will take $K=0.1$ and at the end of the paper, we will comment on the consequences of considering different values of $K$. Finally we will denote by $h$ the constant value of the Hamiltonian $H=h$ over each solution and will be called the energy.

The equations of the motion of the CP problem associated with (2) are

$$
\left\{\begin{aligned}
\dot{x} & =p_{x}+y \\
\dot{y} & =p_{y}-x \\
\dot{p}_{x} & =p_{y}-\frac{x}{r^{3}}-K, \\
\dot{p}_{y} & =-p_{x}-\frac{y}{r^{3}}
\end{aligned}\right.
$$

with ${ }^{\cdot}=\frac{d}{d t}$ and they satisfy the symmetry

$$
\left(t, x, y, p_{x}, p_{y}\right) \longrightarrow\left(-t, x,-y,-p_{x}, p_{y}\right)
$$

This implies that, for each solution of the equations of motion, there also exists another one which is symmetric with respect to $y=0$ in the configuration space $(x, y)$.
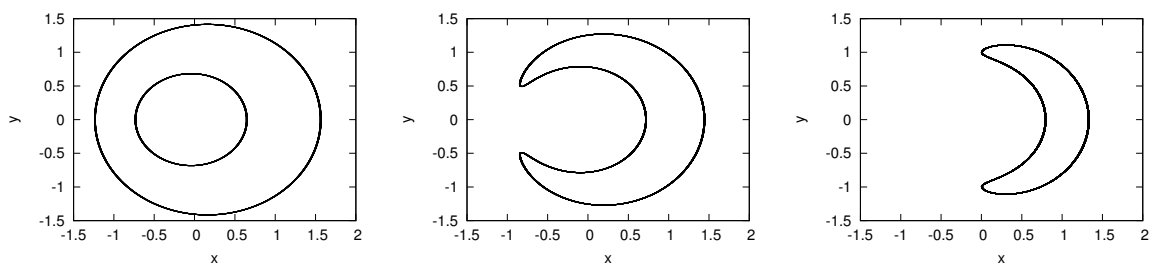

Figure 1: Zero velocity curves in $(x, y)$ coordinates for $K=0.1$ and the following energies: $h=-1.7<h_{1}$ (left), $h=-1.585 \in\left(h_{1}, h_{2}\right)$ (middle) and $h=-1.4 \in\left(h_{1}, h_{2}\right)$ (right). 
The CP problem in rotating coordinates has two equilibrium points, denoted by $L_{1}$ and $L_{2}$ and located in the $x$-axis at $x<0$ and $x>0$ respectively. Taking into account the eigenvalues of the Jacobian matrix of the vector field 95 (3) at the equilibrium points, it can be proved that $L_{1}$ is of type saddle $\times$ center for any value of $K$, whereas $L_{2}$ is of type center $\times$ center for $K$ for $K \leq \frac{3^{-4 / 3}}{2} \simeq 0.11556021$ and is a complex saddle for $K>\frac{3^{-4 / 3}}{2}$ (see [2]). We denote by $h_{i}, i=1,2$ the energy at each equilibrium point.

Concerning the existence of periodic orbits (PO), just applying the well

known Lyapunov theorem (see for example [13]), we claim that, for any $K>$ 0 each equilibrium point gives rise to families of periodic orbits, with $h$ as parameter, the so called Lyapunov periodic orbits (LPO). More precisely, there exists:

- a family of LPO around $L_{1}$ (denoted by LPO1), for $h>h_{1}$ which are unstable (at least for $h$ close to $h_{1}$ ). The associated invariant stable/unstable manifolds play a key role as will be shown later on.

- A family of LPO of long period around $L_{2}$ (LPO2l): it exists for $h_{1}<h \leq$ $h_{2}$ and the orbits are stable.

- A family of LPO of short period around $L_{2}$ (LPO2s): it exists for $h \geq h_{2}$ and the stability of the orbit depends on the value of the Hamiltonian $h$.

We also recall the Hill's regions (following the naming in Celestial Mechanics), that is, the regions in configuration space $(x, y)$ where the motion is possible. We plot in Figure 1 the boundary curves of such regions, called the zero velocity curves (zvc). In particular, the motion is forbidden in the ring region (between the two closed curves) for $h<h_{1}$ (Figure 1 left), and inside the moon shaped region for $h_{1}<h<h_{2}$. For the purposes of this paper, the orbits that go far from the origin and come close to it will take place necessarily in the bounded inner component for $h<h_{1}$. For $h_{1}<h<h_{2}$, such to and from motion can take place both in the inner region and in the outer region through the bottleneck around the equilibrium point $L_{1}$ where the LPO1 and the invari- 
ant manifolds are located and the mixing of such orbits becomes dramatic (see Section 4). Finally, the motion is possible everywhere for $h \geq h_{2}$.

\section{Ejection/collision orbits. Methodology}

In this Section we explain the methodology used to deal with the ECO.

${ }_{125}$ We sketch the necessary changes of variables to regularize the singularity at the origin, we describe the collision manifold (obtained when $r=0$ from the outcoming differential equations) and we account for the different strategies to study $n$-ECO, both as individual orbits and from a global point of view.

\subsection{Regularization of the singularity at the origin}

Our goal is to study orbits that not only pass close to the origin but even that collide with (or eject from) it. So a first step is to remove the singularity $r=0$ from the equations of motion (3).

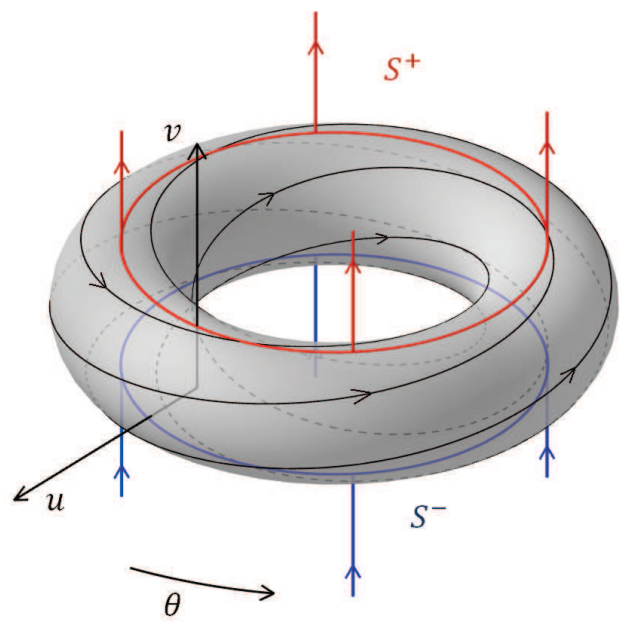

Figure 2: Collision manifold

We introduce the canonical change of polar coordinates

$$
\begin{array}{ll}
x=r \cos \theta & p_{x}=p_{r} \cos \theta-\frac{p_{\theta}}{r} \sin \theta \\
y=r \sin \theta & p_{y}=p_{r} \sin \theta+\frac{p_{\theta}}{r} \cos \theta
\end{array}
$$


and the Hamiltonian (2) becomes

$$
H\left(r, \theta, p_{r}, p_{\theta}\right)=\frac{1}{2}\left(p_{r}^{2}+\frac{p_{\theta}^{2}}{r^{2}}\right)-p_{\theta}-\frac{1}{r}+K r \cos \theta
$$

The associated Hamiltonian system of ODE is

$$
\left\{\begin{aligned}
\dot{r} & =p_{r} \\
\dot{\theta} & =\frac{p_{\theta}}{r^{2}}-1 \\
\dot{p}_{r} & =\frac{p_{\theta}^{2}}{r^{3}}-\frac{1}{r^{2}}-K \cos \theta \\
\dot{p}_{\theta} & =K r \sin \theta
\end{aligned}\right.
$$

Following McGehee's ideas (see [15] in the context of the elliptic RTBP), we introduce the new variables

$$
v=\dot{r} r^{1 / 2} \quad u=r^{3 / 2} \dot{\theta}
$$

and a change of time $d t / d \tau=r^{3 / 2}$, such that the system of ODE becomes

$$
\left\{\begin{array}{l}
r^{\prime}=v r \\
\theta^{\prime}=u \\
v^{\prime}=\frac{1}{2} v^{2}+u^{2}-1+2 u r^{3 / 2}+r^{3}-K r^{2} \cos \theta \\
u^{\prime}=-\frac{1}{2} u v-2 v r^{3 / 2}+K r^{2} \sin \theta
\end{array}\right.
$$

where $^{\prime}=d / d \tau$. We remark that the singularity $r=0$ has been removed. If we consider the hamiltonian (5) in these variables, which is a first integral, the relation $H=h$ becomes

$$
0=-r h+\frac{1}{2}\left(v^{2}+u^{2}\right)-\frac{1}{2} r^{3}-1+K r^{2} \cos \theta
$$

Finally we notice that the equations (8) satisfy the symmetry

$$
(\tau, r, \theta, v, u) \quad \longrightarrow(-\tau, r,-\theta,-v, u)
$$

\subsection{The collision manifold}

For each energy level of the constant Hamiltonian, system (8) has an invariant manifold $\Lambda$ defined by $r=0$, called the collision manifold (see also the 
same kind of study for the Restricted three-body problem in [14]). From (9) we conclude that $\Lambda$ is a torus, that is,

$$
\Lambda=\left\{u^{2}+v^{2}=2, \quad \theta \in[0,2 \pi]\right\}
$$

and the dynamics on this torus is given by

$$
\left\{\begin{array}{l}
\theta^{\prime}=u \\
v^{\prime}=\frac{1}{2} v^{2}+u^{2}-1 \\
u^{\prime}=-\frac{1}{2} u v .
\end{array}\right.
$$

In order to show the orbits of the flow on this torus, in Figure 2 (imported from [14]), we plot the torus taking the coordinates in the plane $(u, v)$ for each $\theta$, and varying $\theta \in[0,2 \pi]$. Due to the first equation in system (12), $\theta(\tau)$ increases (decreases) for $u(\tau)>0(<0)$, when varying $\tau$. Concerning the second equation, from the relation (11) we obtain $v^{\prime}=\frac{u^{2}}{2}$, so $v(\tau)$ is an increasing function in $\tau$. On the other hand, system (8) has two circumferences of equilibrium points, belonging to $\Lambda$, defined by $S^{+}=\{r=0, \theta, v=\sqrt{2}, u=0, \theta \in[0,2 \pi]\}$ and $S^{-}=\{r=0, \theta, v=-\sqrt{2}, u=0, \theta \in[0,2 \pi]\}$. We can clearly see these two circumferences in Figure 2 and the increasing character of $v$ and the monotone behavior of $\theta$ as well.

In order to determine the stability of the equilibrium points, we consider the linearization of system (8) at the corresponding equilibrium points belonging to $S^{ \pm}$. We define the matrix $M^{ \pm}$as

$$
M^{ \pm}=\left(\begin{array}{cccc} 
\pm \sqrt{2} & 0 & 0 & 0 \\
0 & 0 & 0 & 1 \\
0 & 0 & \sqrt{2} & 0 \\
0 & 0 & 0 & \mp \sqrt{2} / 2
\end{array}\right),
$$

The matrix $M^{+}$has eigenvalues:

$$
\lambda_{1}=-\sqrt{2} / 2, \lambda_{2}=\lambda_{3}=\sqrt{2}, \lambda_{4}=0
$$


and corresponding eigenvectors:

$$
\boldsymbol{v}_{1}=(0,-\sqrt{2}, 0,1), \boldsymbol{v}_{2}=(0,0,1,0), \boldsymbol{v}_{3}=(1,0,0,0), \boldsymbol{v}_{4}=(0,1,0,0) .
$$

Therefore each equilibrium point $P \in S^{+}$has an associated 2-d unstable manifold $W^{u}(P)$ and a 1-d stable one $W^{s}(P)$. Similarly, each equilibrium point $Q \in S^{-}$has a 2-d stable manifold $W^{s}(Q)$ and a 1-d unstable one $W^{u}(Q)$.
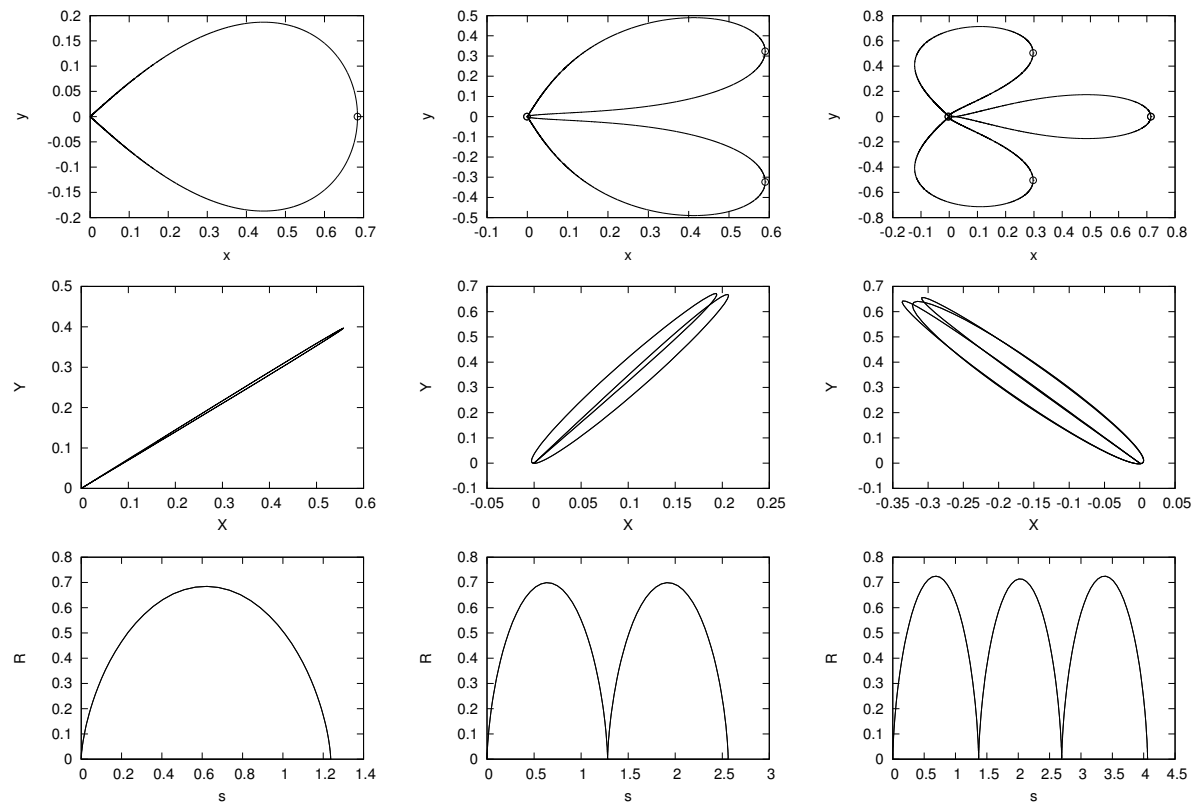

Figure 3: $K=0.1, h=-1.4$. Top. Examples of 1, 2 and 3-ECO (left, middle and right respectively) in configuration space rotating rescaled variables $(x, y)$. The round points satisfy $\dot{x}=0, \dot{y}<0$. Middle. The ECO in original (non-rotating) coordinates $(X, Y)$. Bottom. The ECO in $(s, R)$ variables (right), $s$ the physical time and $R$ the distance to the origin.

We can conclude that any orbit ejecting from (colliding with) the origin is precisely an orbit belonging to $W^{u}(P)\left(W^{s}(Q)\right)$ for some equilibrium point $P \in S^{+}\left(Q \in S^{-}\right)$. More particularly, any ejection-collision orbit (ECO) is a heteroclinic orbit connecting some $P$ (when $\tau \rightarrow-\infty$ ) with some $Q$ (when $\tau \rightarrow+\infty)$.

Finally, we remark that when $K=0$, we obtain the Kepler problem, and it 
is well known that, for $h<0, W^{u}\left(S^{+}\right)$and $W^{s}\left(S^{-}\right)$coincide, i. e., any ejection orbit is also a collision one. The interesting point here is that for $K \neq 0, W^{u}\left(S^{+}\right)$ and $W^{s}\left(S^{-}\right)$do not coincide anymore and there appears a rich dynamics.
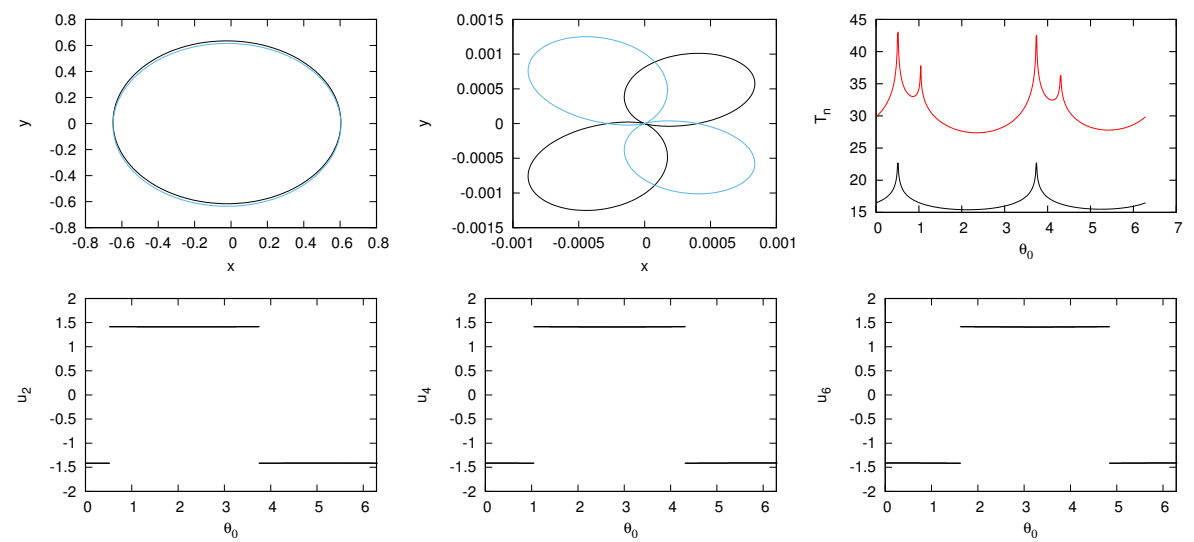

Figure 4: $K=0.1, h=-1.6$. Top. $D_{n}^{+}$(in black) and $D_{n}^{-}$(in blue) in (x,y) variables: $n=1$ (left), $n=2$ (middle). Right. Curves $\left(\theta_{0}, T_{n}\right)$ for $n=2$ (black) and $n=4$ (red). Bottom. Curve $\left(\theta_{0}, u_{n}\right)$, for $n=2$ (left), $n=4$ (middle) and $n=6$ (right).

\section{3. n-Ejection-collision orbits}

We recall, from the introduction, that an $n$-ejection-collision orbit ( $n$-ECO), is a trajectory that ejects from the origin, reaches $2 n-1$ relative extrema in the distance $r$, typically $n$ maxima and $n-1$ minima, before colliding with the origin. In Figure 3, for $K=0.1$ and $h=-1.4$, we show an example of 1,2 and 3 -ECO in rotating rescaled variables $(x, y)$ (top), in original non-rotating variables $(X, Y)$ (middle), and in $(s, R)$ variables ( $s$ the physical time and $R$ the distance to the origin) for the particular value $w=1, F=K=0.1$. Of course the range for the rescaled time $\tau$ would be $(-\infty, \infty)$ for any of them. Along the paper we will typically study the CP using the rotating rescaled variables $(x, y)$ and the rescaled time $\tau$.

We aim at studying orbits that go far from and come close to the origin several times. So we can regard the $n$-ECO simply as the skeleton of such orbits. In this subsection we will describe the numerical machinery to study the 
$n$-ECO and later on we will show the consequences that such orbits have on the to and fro motion.

\subsubsection{Computation of $n-E C O$}

Fixed a value of $K>0$ and $H=h$, let us describe first how to compute ejection orbits, that is orbits on $W^{u}\left(S^{+}\right)$. To do so, we take an equilibrium point $P=\left(0, \theta_{0}, \sqrt{2}, 0\right), \theta_{0} \in[0,2 \pi]$, and consider the tangent plane to $W^{u}(P)$, through $P$, generated by the eigenvectors $\boldsymbol{v}_{2}$ and $\boldsymbol{v}_{3}$ (of matrix $M^{+}$), i.e. vectors

like $\boldsymbol{v}=(\beta, 0, \gamma, 0)$, with $\beta, \gamma \in \mathbb{R}$. The restriction that the orbit $W^{u}(P)$ also belongs to $H=h$ can be written as the perpendicularity condition between vector $\boldsymbol{v}$ and $\boldsymbol{n}=(-h, 0, \sqrt{2}, 0)$, which is the gradient vector to the energy level set $H=h$ defined implicitly by (9). So we require the condition

$$
-\beta h+\gamma \sqrt{2}=0
$$

and we take the initial condition of an ejection orbit associated with the point $P=\left(0, \theta_{0}, \sqrt{2}, 0\right)$ as

$$
\left(0, \theta_{0}, \sqrt{2}, 0\right)+s \frac{\boldsymbol{w}}{\|\boldsymbol{w}\|}
$$

with $\boldsymbol{w}=(1,0, h / \sqrt{2}, 0)$ and $s>0$ a small quantity (typically ranging from $19010^{-7}$ to $10^{-5}$ ). We will say that the ejection orbit is defined by $\theta_{0}$ (its initial condition determined from $\left.\theta_{0}\right)$. Varying $\theta_{0} \in[0,2 \pi]$, we generate a set of initial conditions belonging to the (tangent plane to the) unstable manifold $W^{u}\left(S^{+}\right)$ for $H=h$ fixed. For each initial condition we integrate the system (8) forward in time and check that the Hamiltonian remains constant (through the relation (9)) along the integration.

We proceed similarly in order to obtain a set of initial conditions of the collision orbits belonging to the stable manifold $W^{s}\left(S^{-}\right)$for $H=h$ fixed. For each initial condition, we integrate system (8) backward in time and check the relation (9). 

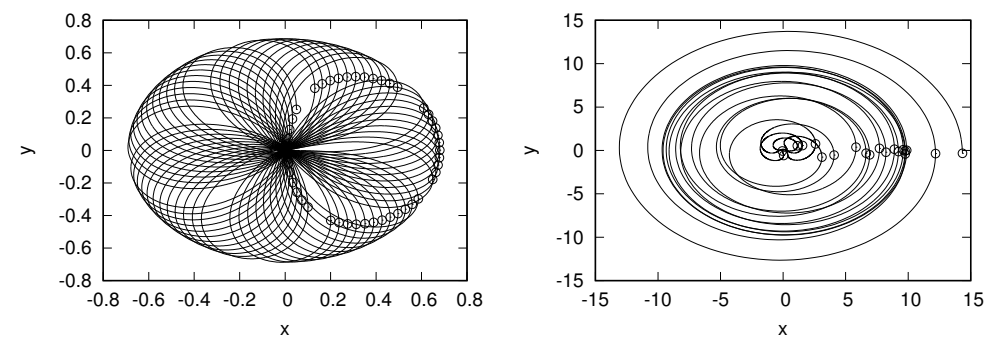

Figure 5: $K=0.1$ and $(x, y)$ coordinates. Ejection orbit and the associated points in $\hat{\Sigma}$ -round points-. A bounded one (left) for $h=-1.4$, an escape one (right) for $h=-0.8$.

The next goal is to compute $n$-ECO, for a given $n$.

A very useful numerical tool will be to consider the Poincaré section $\Sigma$ : $g(\boldsymbol{x})=g(r, \theta, v, u)=v=0$, which corresponds to a passage of maximum or minimum distance to the origin (if $r \neq 0$ ). Furthermore, given $K$ and $H=h$, let us denote $\Sigma_{n}$, the $n$-th crossing with $\Sigma$ and define $D_{n}^{+}=W^{u}\left(S^{+}\right) \cap \Sigma_{n}$, and $D_{n}^{-}=W^{s}\left(S^{-}\right) \cap \Sigma_{n}$. For example, in Figure 4 top left, we show the curves $D_{1}^{+}$ (in black) and $D_{1}^{-}$(in blue). We also define $T_{n}\left(\theta_{0}\right)$ and $u_{n}\left(\theta_{0}\right)$ as the values of $\tau$ and $u$ for an orbit on $W^{u}\left(S^{+}\right)$(defined by $\theta_{0}$ ) when it reaches $\Sigma_{n}$. We will drop the $\theta_{0}$ notation and we will simply write $T_{n}$ and $u_{n}$. See the curves $\left(\theta_{0}, T_{2}\right)$ and $\left(\theta_{0}, u_{n}\right)$, for $n=2,4,6$ in Figure 4.

In the procedure to compute $n$-ECO we will distinguish two steps.

First step. Existence of $n$-ECO. We want to visualize if there exist such orbits for a given $K$ and $H=h$. To do so we consider different approaches:

- First strategy, we look for intersection points belonging to $D_{n}^{+} \cap D_{n}^{-}$. Any such point corresponds to an $n$-EC orbit. For example, Figure 4 top left shows the curves $D_{1}^{+}$and $D_{1}^{-}$and we see there are two intersecting points that belong to $D_{1}^{+} \cap D_{1}^{-}$. Of course, due to the symmetry (10), we expect to have at least two 1-ECO, which correspond to the intersecting points on the $x$ axis. Such two ECO will be symmetric with respect to the $x$ axis (in the $(x, y)$ projection). See for example Figure 3 left. The outstanding fact is that there are no other 1-ECO (for small values of $h$ ) as happens in other problems (there appear typically four 1-ECO for small values of 
the energy and any value of the mass parameter in the Restricted threebody problem, see [14]). We remark that for $n \geq 2$, the curves $D_{n}^{+}$and $D_{n}^{-}$become more involved and a more detailed analysis is required to determine the number of $n$-ECO. See, for example, the curves $D_{n}^{+}$and $D_{n}^{-}$, for $n=2$ in Figure 4 top.

- A second strategy is based on the property that $n$-ECO are heteroclinic orbits, so any $n$-ECO defined by a suitable $\theta_{0}$ (for its initial condition) ejected from the origin (when $\tau \rightarrow-\infty$ ), will take an infinite time $\tau$ to reach the origin again after $2 n-1$ crossings with the section $\Sigma$. So we just need to plot the curve $\left(\theta_{0}, T_{2 n}\right)$, and look at the vertical asymptotes in time. From now on any value $\theta_{0}$ such that $T_{2 n}$ becomes infinite will be called a singularity. For example in Figure 4 top right, from the curve $\left(\theta_{0}, T_{2}\right)$ (in black) we can see that there are two singularities, in accordance with the previous strategy. To look for 2 -ECO, we plot $\left(\theta_{0}, T_{4}\right)$ (in red) in Figure 4 top right, and of course there appear four asymptotes: two of them correspond to the 1-ECO (for the associated values of $\theta_{0}, T_{4}$ should not be computed but we take a discrete grid of values of $\theta$ and the exact values of $\theta_{0}$ for 1 -ECO are not considered) and two new ones corresponding to the 2-ECO. So we remark that, in general, for $n \geq 2$ we must compare the curve $\left(\theta_{0}, T_{2 n}\right)$ with the $\left(\theta_{0}, T_{2 n-2}\right)$ one in order to identify the new vertical asymptotes in $T_{2 n}$ and that do not appear in the curve $\left(\theta_{0}, T_{2 n-2}\right)$. Those will correspond to the $n$-ECO specifically.

- Finally, a third strategy has to do with the geometry of the ECO itself. More precisely, a typical $n$-ECO (defined by $\theta_{0}$-which determines its initial condition-) may be regarded as a heteroclinic orbit that behaves like this: it ejects from collision in physical (non-regularized) coordinates, this means that in regularized variables tends to a particular equilibrium point $P \in \Lambda$ (as $\tau \rightarrow-\infty)$ with $r=0, u=0, v=\sqrt{2}$ and some particular $\theta_{0}$; as time goes on, it has $n$ far passages from the origin and $n-1$ close to it (that is $2 n-1$ intersections with $\Sigma$, i. e. $v=0$ ) before final 
collision -in physical variables- for a finite physical time $s$, this means that the trajectory tends to a suitable equilibrium point $Q \in \Lambda$, with $r=0, u=0, v=-\sqrt{2}$ and some suitable $\theta$ as $\tau \rightarrow \infty$ (in regularized variables). In particular, if we take two other ejection orbits, called $E_{1}$ and $E_{2}$ (which are not $n$-ECO), $E_{1}$ defined by $\theta_{0,1}<\theta_{0}$ and $E_{2}$ by $\theta_{0,2}>\theta_{0}$, both $\theta_{0,1}, \theta_{0,2}$ very close to $\theta_{0}$, the corresponding trajectories will follow a path very close to the heteroclinic orbit. However, after the $2 n-1$ intersections with $\Sigma$, the trajectory $E_{1}\left(E_{2}\right)$ will go on passing very close to the point $Q \in \Lambda$, with $v$ close to $-\sqrt{2}$ but, due to the flow behavior on $\Lambda$ (see Figure 2), the trajectory $E_{1}\left(E_{2}\right)$ will follow near $\Lambda$ with increasing values of $v$ with $u>0(u<0)$ until $v=0$, that is until the $2 n$ crossing with $\Sigma$ is reached. (Notice that it might happen the other way around $u<0$ for $E_{1}\left(u>0\right.$ for $\left.\left.E_{2}\right)\right)$. In particular, for this $2 n$-th crossing the value of $u_{2 n}$ will be very close to + or $-\sqrt{2}$ (since at $\Lambda$, when $v=0$, $u= \pm \sqrt{2})$. Therefore a discontinuity or jump in the curve $\left(\theta_{0}, u_{2 n}\right)$ from $u<0$ to $u>0$ (or viceversa) provides the numerical existence of a suitable $\theta_{0}$ of a heteroclinic orbit, that corresponds to a $n$-ECO. So, similarly to the previous strategy, we just need to plot the curve $\left(\theta_{0}, u_{2 n}\right)$ and look for discontinuities in $\theta_{0}$. The advantage of this approach is that the jumps in the curve $\left(\theta_{0}, u_{2 n}\right)$ provide directly the suitable values of $\theta_{0}$ corresponding to $n$-ECO regardless of what happens for smaller values of $n$ (since the curve $\left(\theta_{0}, u_{2 n}\right)$ is generated from a discrete grid of values of $\theta_{0}$ and the exact values of $\theta_{0}$ for which $u_{2 k}, k<n$, is not defined -that correspond to $k$-ECO- are not taken into account). See the curves $\left(\theta_{0}, u_{2 n}\right)$ in Figure 4 for $n=1,2,3$. The two discontinuities $\left(u_{2 n}\right.$ changing sign from positive to negative or viceversa) locate the two values of $\theta_{0}$ of $n$-ECO, for $n=1,2,3$.

We remark that we have applied the third strategy to numerically compute a particular $n$-ECO, simply considering a bisection method taking the $\theta_{0}$ as variable and the value of $u_{2 n}$ as function.

Second step. Continuation of families of $n-E C O$ for a given $K$ and $n$. We 
have applied the second and third strategies doing a massive simulation, varying the energy parameter $h$, and for each $h$, considering the whole set of initial conditions, i.e., for $\theta \in[0,2 \pi]$. See next Section for the results obtained.
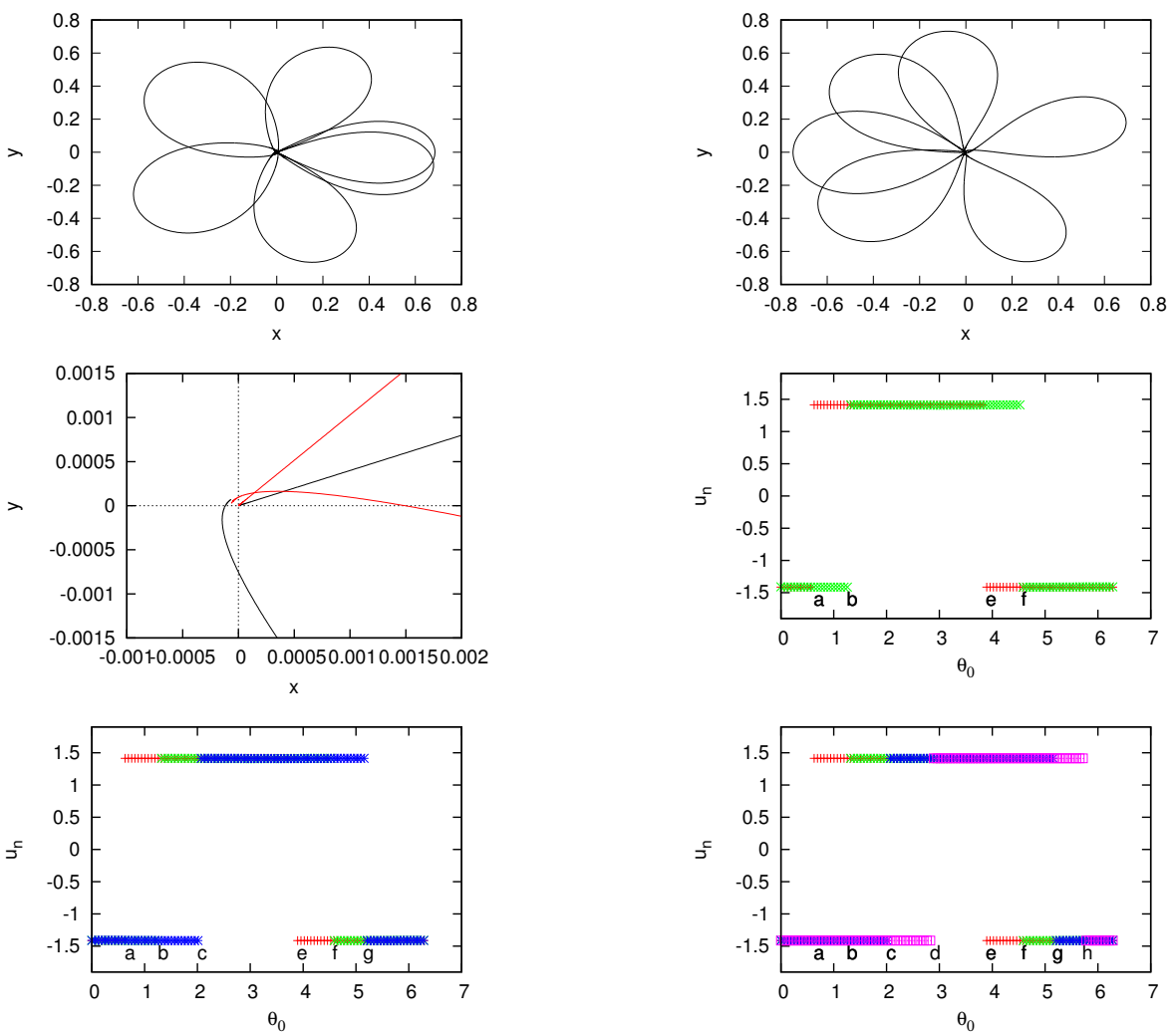

Figure 6: $K=0.1, h=-1.4$. Top. Two different ejection orbits (in $(x, y)$ coordinates). Middle. Left. Two ejection orbits (in $(x, y)$ coordinates), one with a close passage to the origin with $u>0$ (in red), and the other one with $u<0$ (in black). Right. Curves $\left(\theta_{0}, u_{n}\right)$, for $n=2$ (red) and $n=4$ (green). Bottom. Curves $\left(\theta_{0}, u_{n}\right)$, for $n=2,4,6$ (left, $n=6$ in blue) and for $n=2,4,6,8$ (right, $n=8$ in pink).

\subsection{Global behavior of $W^{u, s}\left(S^{+,-}\right)$}

Another approach that becomes useful in order to describe the behavior of the whole set of orbits in $W^{u}\left(S^{+}\right)\left(W^{s}\left(S^{-}\right)\right)$is by means of what we called Poincaré section plots (PSP) in [1]: for $K>0$ and $h$ given, we consider a new Poincaré section $\hat{\Sigma}$, defined by $\dot{x}=0$ and $\dot{y}<0$, and plot the successive crossings 
290

Such plots show how the orbits look like, it they escape or not, and (somewhat) encapsulate their behavior, giving an insight for to and fro motion. In particular, any $n$-ECO will be represented by a finite number of points in $\hat{\Sigma}$. In Figure 3 we show 1, 2, and 3-ECO together with their points in $\hat{\Sigma}$. However, a bounded ejection orbit (which is not an ECO), will typically look like a rosette (see Figure 5 left) or we may also have ejection orbits that escape (Figure 5 right).
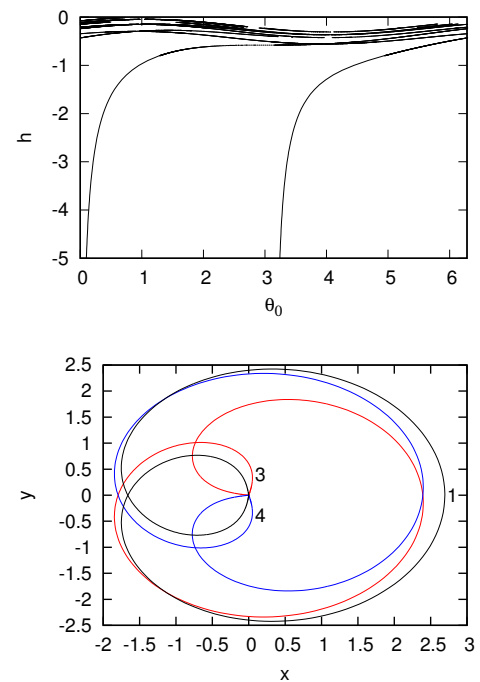

Figure 7: $K=0.1$, Top. Characteristic curves of families of 1-ECO in variables $\left(\theta_{0}, h\right)$. Right. Zoom. Bottom. Symmetric and non-symmetric 1-ECO (in $(x, y)$ variables) labeled by $1,2,2 a, 3,4$ if they belong to families $f 1, f 2, f 2 a, f 3, f 4$ respectively.

\section{To and fro motion. Numerical simulations. Results.}

As stated in the Introduction, the $n$-ECO provide the skeleton of the natural to and fro motion. Actually, once we study such orbits, just by continuity with respect to initial conditions, we can find other orbits with close/far passages to the origin, and which do not belong to $W^{u}\left(S^{+}\right) \cup W^{s}\left(S^{-}\right)$. So this section is devoted to provide the results obtained considering massive simulations of both ECO and ejection/collision orbits. More precisely, we will consider geometrical 
aspects, continuation of families and the discussion of appearing bifurcations.

Finally, we will explain the role that such orbits play on the to and fro motion in the dynamics of the problem from a global point of view.

The simulations have been done taking the value $K=0.1$, for which $h_{1}=$ -1.59836975 and $h_{2}=-1.39829568$. A comment regarding other values of $K$ is done at the end of this Section.
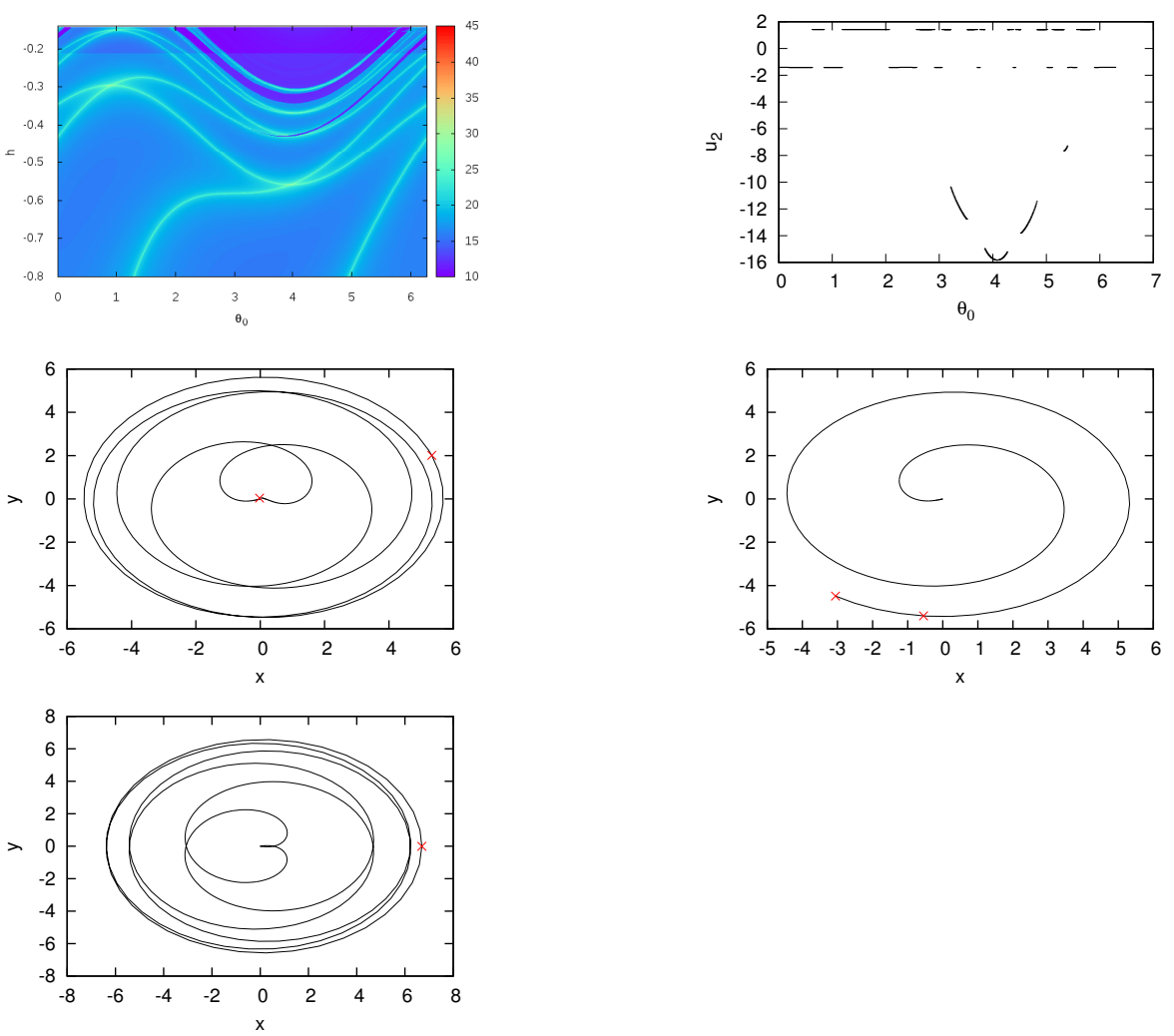

Figure 8: $K=0.1$, Top left. Diagram $\left(\theta_{0}, h, T_{2}\right), \theta_{0}(h)$ in the $x(y)$ axis and $T_{2}$ for the chronogram. For the remaining plots $h=-0.3$. Top right. Curve $\left(\theta_{0}, u_{2}\right)$. Middle. Two ejection orbits (in $(x, y)$ variables) for close values of $\theta_{0}$, the left one has the second crossing close to the origin (which is the typical behaviour) but the right one has the second crossing far from it. Bottom. A 1-ECO with a far first crossing from the origin for $h=-0.05$ 


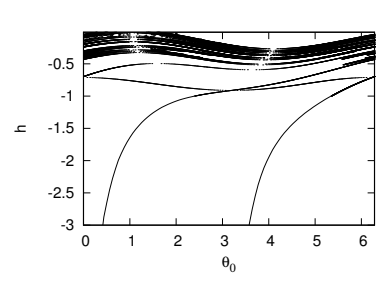

\subsection{Coding the close passages to the origin.}

As mentioned above, the $n$-ECO have a finite number of close and far passages from the origin. However, we may also consider those ejection orbits with infinitely many passages close and far from the origin. Due to the existence of the circumference $S^{-}$of equilibrium points in the collision manifold $\Lambda$, and the internal dynamics on $\Lambda$, there is a huge freedom for any orbit passing close to the origin, i. e., the orbit passes close to an equilibrium point in $S^{-}$, which can be a different point for each close passage. In Figure 6 top we show two different examples of ejection orbits. We see in the plots that the orbits pass close to the origin in an apparent erratic way. So, a natural question that arises is to give a certain code to such close passages. A natural answer is to take into account the sign of $u$ at the close passage, i. e., the sign of $\dot{\theta}$ which decides the increasing/decreasing character of $\theta$.
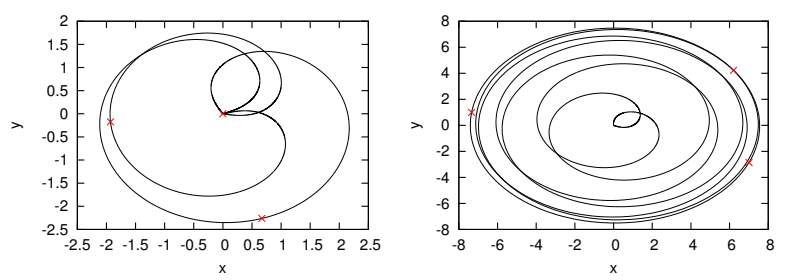

Figure 9: $K=0.1$. Left. Characteristic curves of families of 2-ECO in variables $\left(\theta_{0}, h\right)$. Projection $(x, y)$ of two 2-ECO for $h=-0.43$ (middle) and $h=-0.01$ (right). The cross points in $\Sigma$ are plotted in red.

This is shown, for example, in Figure 6 middle left, where two ejection orbits are plotted, the red (black) one has a close passage with $u>0(u<0)$. The strategy for coding an ejection orbit (that typically behaves describing sequentially far and close passages to the origin) is to encode each close passage to the origin by a 1 or -1 according to the sign of $u$ at that close passage. So an ejection orbit, defined by $\theta_{0}$, has an associated sequence such that the $k$-th element of the sequence is 1 or -1 according to the sign of $u_{2 k}$, sequence that will be finite if the ejection orbit is an ECO. To apply this strategy, we just need to overlap the curves $\left(\theta_{0}, u_{n}\right)$ for even $n$. Let us clarify how it works. 
We take, for example, $K=0.1$ and $h=-1.4$. In Figure 6 middle right we overlap the curves $\left(\theta_{0}, u_{n}\right)$, for $n=2$ (red curve) and $n=4$ (green one). The overlapping defines the following intervals in the variable $\theta_{0}:[0, a]$ where for each $\theta_{0} \in[0, a]$ the ejection orbit defined by that $\theta_{0}$ will satisfy that $u_{2}<0$ and $u_{4}<0 ;[a, b]$ where $u_{2}>0$ and $u_{4}<0 ;[b, e]$ where $u_{2}>0$ and $u_{4}>0 ;[e, f]$ where $u_{2}<0$ and $u_{4}>0$ and $[f, 2 \pi]$ where $u_{2}<0$ and $u_{4}<0$. This means that for each ejection orbit defined by $\theta_{0}$ with $\theta_{0} \in[0, a]$, the first two elements of the associated sequence will be -1 (since $u_{2}<0$ and $u_{4}<0$ ) so the corresponding sequence will be $\{-1,-1, \ldots\}$. Similarly for each ejection orbit with $\theta_{0} \in[a, b]$, the sequence will be $\{1,-1, \ldots\}$ (since $u_{2}>0$ and $u_{4}<0$ ); if $\theta_{0} \in[b, e]$, the sequence will be $\{1,1, \ldots\}$; if $\theta_{0} \in[e, f]$, the sequence will be $\{-1,1, \ldots\}$ and if $\theta_{0} \in[f, 2 \pi]$, the sequence will be $\{-1,-1, \ldots\}$. In Figure 6 bottom left we have overlapped the curves $\left(\theta_{0}, u_{n}\right)$, for $n=2,4,6$ ( $n=6$ in blue), so we may infer the third element of the sequence of each ejection orbit. And in Figure 6 bottom right the overlapping of the curves $n=2,4,6,8$ ( $n=8$ in pink) is shown. So, from this overlapping and according to the sign of $u_{n}$ for $n=2,4,6,8$, each ejection orbit, defined by $\theta_{0}$, has the following coding sequence (we know its first four elements)

- for $\theta_{0} \in[0, a] \cup[h, 2 \pi], \quad\{-1,-1,-1,-1, \ldots\}$

- for $\theta_{0} \in[a, b], \quad\{1,-1,-1,-1, \ldots\}$

- for $\theta_{0} \in[b, c], \quad\{1,1,-1,-1, \ldots\}$

- for $\theta_{0} \in[c, d], \quad\{1,1,1,-1, \ldots\}$

- for $\theta_{0} \in[d, e], \quad\{1,1,1,1, \ldots\}$

355

- for $\theta_{0} \in[e, f], \quad\{-1,1,1,1, \ldots\}$

- for $\theta_{0} \in[f, g], \quad\{-1,-1,1,1, \ldots\}$

- for $\theta_{0} \in[g, h], \quad\{-1,-1,-1,1, \ldots\}$ 
So we can summarize that the successive values of $u$ at the close passages provide geometrical information of such near passages and a code for each ejection orbit.
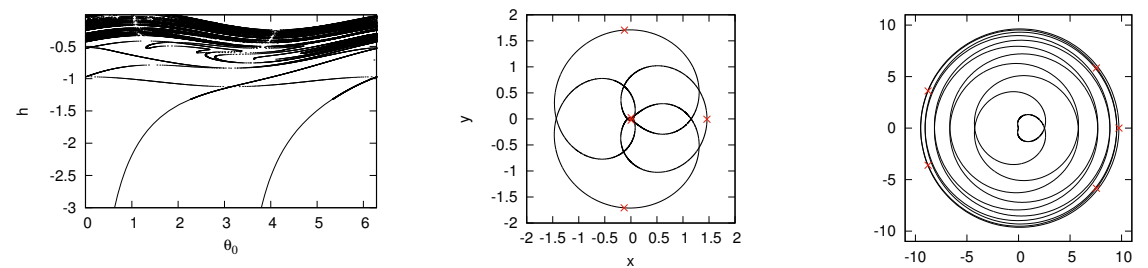

Figure 10: $K=0.1$. Left. Characteristic curves of families of 3-ECO in variables $\left(\theta_{0}, h\right)$. Projection $(x, y)$ of two 3 -ECO for $h=-0.38$ (middle) and $h=-0.012$ (right). The cross points in $\Sigma$ are plotted in red.

\subsection{Families of n-ECO. Bifurcations.}

The purpose of this subsection is to show families of $n$-ECO for $K$ fixed and varying the $h$ parameter. Following the third strategy explained above, we will obtain characteristic curves in variables $\left(\theta_{0}, h\right)$, i. e., curves of points such that each point gives rise to an initial condition of an $n$-ECO, for $n$ given.

So, we now fix a (small) value of $K>0$, and we know that for each $H=h$ with $h<h_{1}$, the $n$-ECO exist on the inner bounded component of the Hill's region (see Figure 1). More particularly, there exist two $n$-ECO. We have done the continuation of such ECO varying $h$ (for ranges of $h$ up to values bigger than $h_{2}>h_{1}$ ) giving rise to what we call the main families $f 1$ and $f 2$.

- Concerning the families of 1-ECO, we show in Figure 7 top, the characteristic curves $\left(\theta_{0}, h\right)$ for $K=0.1$ and $h \in(-5,0)$, of the families $f 1$ and $f 2$. For the sake of comparison, we also show in Figure 8 top left, the diagram $\left(\theta_{0}, h, T_{2}\right)$, where we plot $T_{2 n}$ as a function of $\theta_{0}$ and $h$. The accordance between both plots is apparent. Of course in the last one, we do not obtain unbounded values of $T_{2}$, but finite ones (due to numerical simulations), but the existence of the different families is clearly visible (from the bigger values of $T_{2}$ ). 

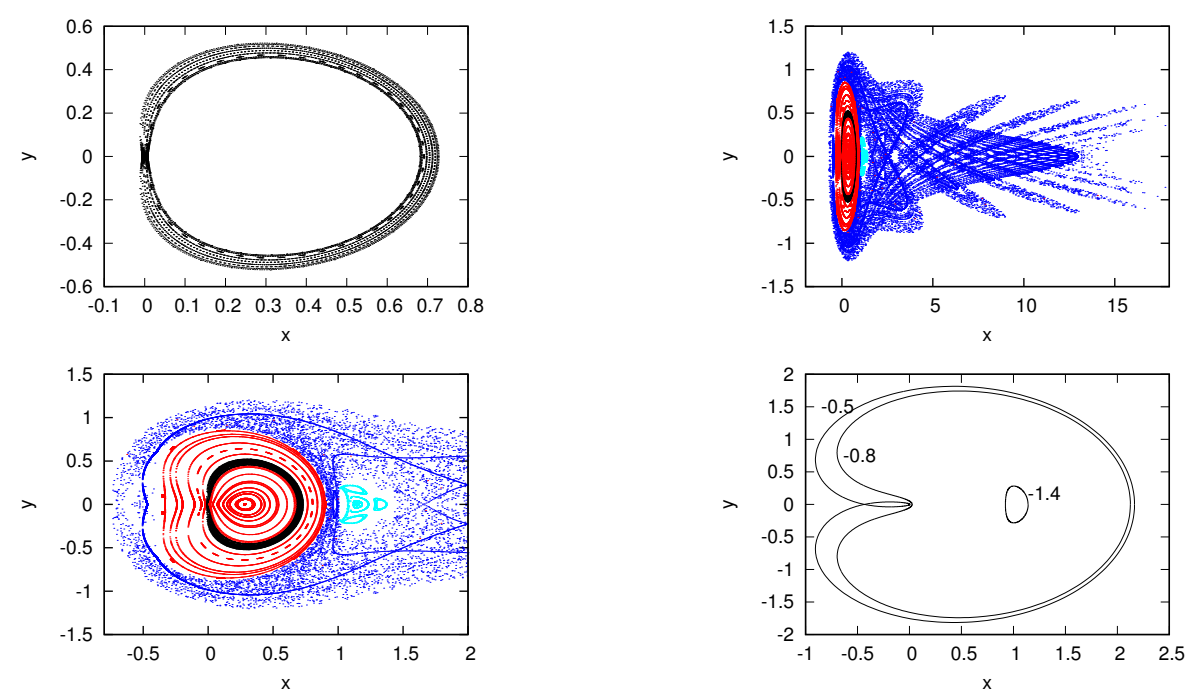

Figure 11: $K=0.1, h=-1.4 \in\left(h_{1}, h_{2}\right)$ and $(x, y)$ coordinates. Top left. PSP of the $W^{u}\left(S^{+}\right)$. Top right. PSP of $W^{u}\left(S^{+}\right)$(in black), the manifolds of LPO1 (in dark blue), some invariant curves (in red) and the stable LPO2s and surrounding invariant curves (in light blue). Bottom. Zoom Right. Lyapunov periodic orbits for $h=-1.4,-0.8,-0.5$.

Three remarks must be made:

First, when $h$ is small, only the families $f 1$ and $f 2$ exist and the associated 1-ECO are symmetric (with respect to the $x$ axis, in the configuration plane $(x, y))$. However, as far as $h$ increases, there appear some bifurcations. We show for example in Figure 7 top right the bifurcated families $f 3$ and $f 4$ from family $f 1$. Such families contain non-symmetric 1-ECO. See in Figure 7 bottom left, the orbit (in black) that belongs to $f 1$ and the bifurcated ones that belong to families $f 3$ (in red) and $f 4$ in blue, for $h=-0.5$. Similarly, families $f 3$ and $f 4$ bifurcate from family $f 2$. For $h=-0.3$, we plot the corresponding orbits: the non-bifurcated symetric ones in family $f 2$ (in black) (and $f 2 a$-in blue- after the bifurcation) and the bifurcated non-symmetric ones in families $f 3$ (in red) and $f 4$ (in pink). We notice that, as $h$ increases, there appear other bifurcations.

The second remark deals with the continuation of the bifurcated families. We can see in Figure 7 top right that there are some missing pieces of the 
characteristic curves. The reason for that is shown in Figure 8. When plotting the curve $\left(\theta_{0}, u_{2}\right)$ we observe the typical behavior of $u_{2}$ (close to the values $395 \pm \sqrt{2}$ ) associated with close passages to the origin at the second crossing with $\Sigma$. However there appear four misplaced pieces of negative values of $u_{2}$ which correspond to the second passage of the orbit with $\Sigma$ but far from the origin. We plot two example orbits (together with the crossing points with $\Sigma$-in red-) in Figure 8 middle: the typical orbit (on the left) with the second crossing close to the origin, but the weird one (on the right) with the second crossing far from it. This weird behaviour is clearly seen in the diagram in the top left Figure 8: the specially dark regions and the thin dark tongue correspond to small values of $T_{2}$ compared to the remaining regions.

Finally the third remark is related with the geometry of the 1-ECO themselves. For small values of $h$, the 1-ECO are simple and reach small values for the maximum distance in $r$ due to the restriction of the Hill's region. See Figure 3 left. However, as $h$ is close to zero, there is no restriction concerning Hill's regions, moreover, we have 1-EC orbits that spiral, on and on, around the origin, reaching big values for maximum $r$. See for example Figure 8 bottom.

- Concerning the continuation of families of 2-ECO and 3-ECO, we show in Figure 9 and 10 the corresponding characteristic curves and bifurcated families. In both cases, the two main families are visible and the pattern of bifurcation is similar.

A remarkable comment, compared with the $1-\mathrm{ECO}$, is that the (unique) 415 point in $\Sigma$, i. e. $v=0$, for $1-\mathrm{ECO}$, corresponds to the furthest passage to the origin. However, for $n$-ECO, $n \geq 2$, the orbit has $2 n-1$ crossings with $v=0$; typically, for small values of $h$, odd and even crossings with $\Sigma$ correspond to far and close passages, respectively. These are the typical $n$-ECO. See Figure 3 middle and right for $n=2$ and $n=3$, respectively. But, for higher values of $h$, ${ }_{420}$ it may happen that the $2 n-1$ crossings with $\Sigma$ take place far from the origin. See Figures 9 and 10 right. 

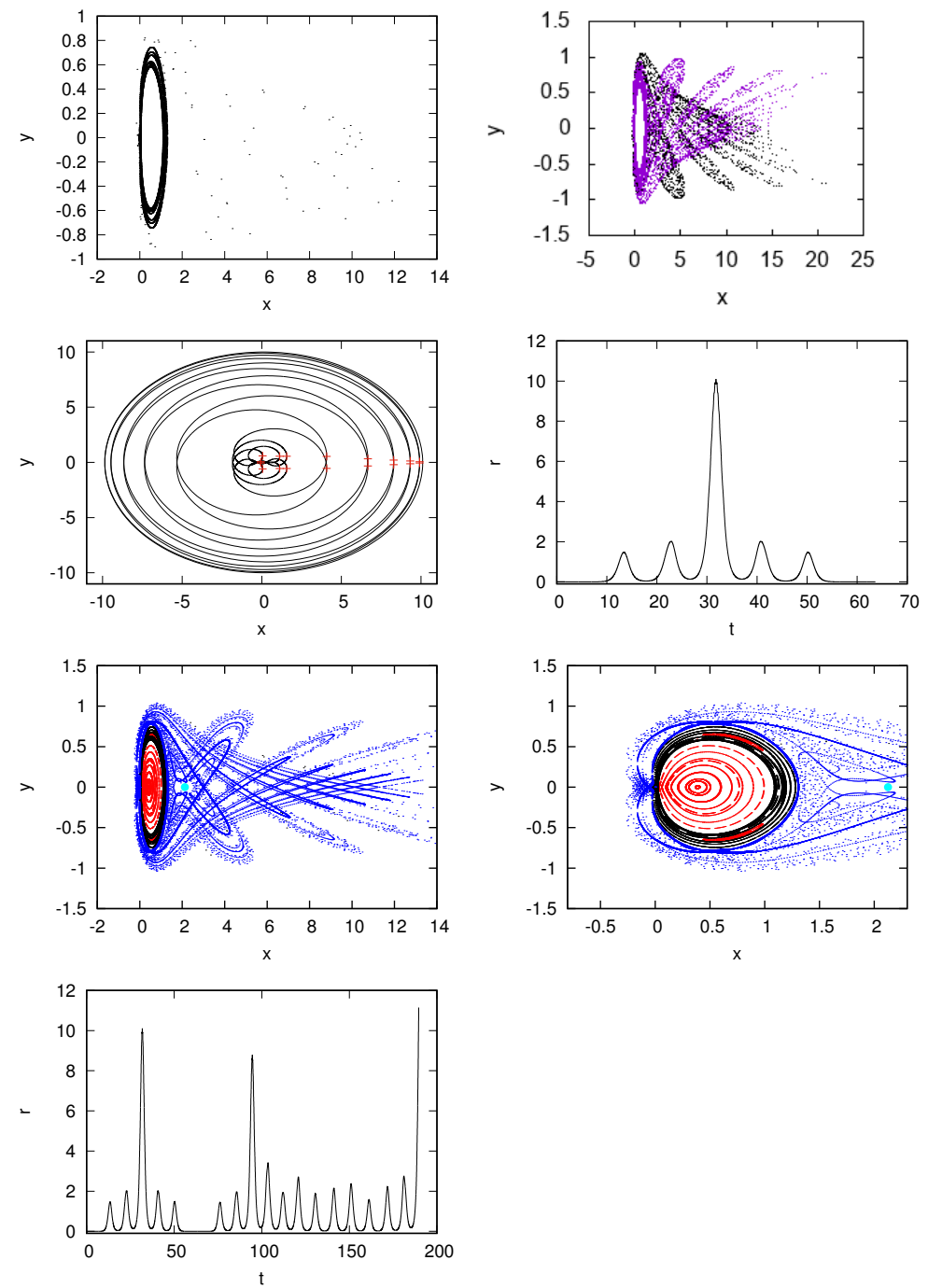

Figure 12: $K=0.1$ and $h=-0.8>h_{2}$. First row. PSP obtained from $W^{u}\left(S^{+}\right)$(left) and from $W^{u}\left(S^{+}\right)$(in black) and $W^{s}\left(S^{-}\right)$(in violet) for a higher number of iterates (right), in $(x, y)$ variables. Second row. ECO in $(x, y)$ variables (left) and in $(\tau, r)$ ones (right). Third row. PSP of $W^{u}\left(S^{+}\right)$(in black), the manifolds of LPO1 (in dark blue), some invariant curves (in red) and the unstable LPO2s (in light blqq). Right. Zoom. Fourth row. Ejection orbit $((\tau, r)$ variables $)$ with erratic to and fro motion that finally ionizes. 

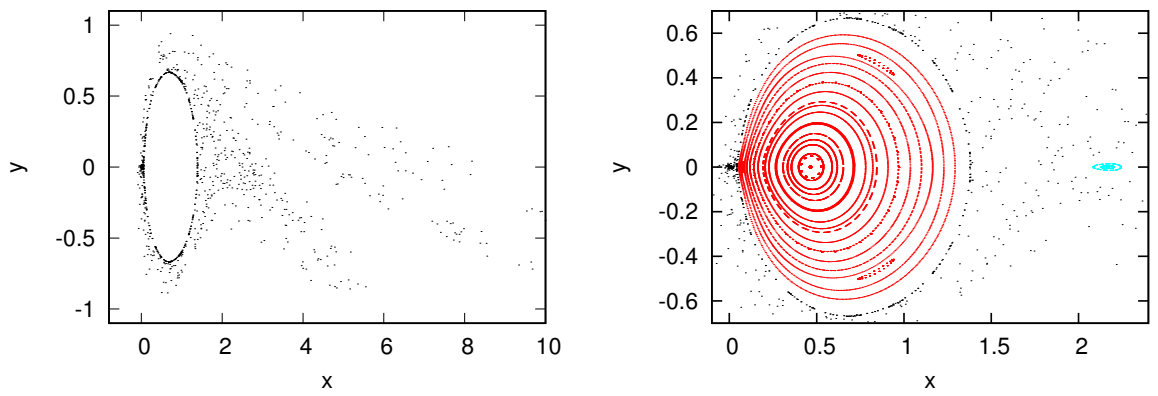

Figure 13: $K=0.1, h=-0.5$. Left. PSP in $(x, y)$ variables of $W^{u}\left(S^{+}\right)$. Right. Zoom including $W^{u}\left(S^{+}\right)$(in black), the inner invariant curves (in red) and the stable LPO2s and the associated surrounding invariant curves (in light blue).

So the skeleton of the to and from motion is qualitatively different: whereas for the typical $n$-ECO, the orbits have alternate very far/very close passages to the origin -the intrinsic definition of to and fro motion-, for the non-typical ones, they only have far passages from the origin before going to collision with the origin.

\subsection{Global dynamics. Poincaré section plots}

So far we have considered the skeleton of to and from motion just taking into account the ejection, collision and ECO up to a (small) number of crossings with the Poincaré section $\Sigma$. In particular, for $K>0$ and $h$ given, just individual orbits have been taken. In this section we want to consider the unstable and stable manifolds manifolds, $W^{u}\left(S^{+}\right)$and $W^{s}\left(S^{-}\right)$, that is the set of ejection/collision as a whole set and its effect on the global dynamics of the system.

A useful approach to do so is, for $K>0$ and $h$ given, to consider the PSP defined in Section 3, that is to save a high number of the crossings -iterates- of any orbit belonging to $W^{u}\left(S^{+}\right)\left(W^{s}\left(S^{-}\right)\right)$with the Poincaré section $\hat{\Sigma}$ defined by $\dot{x}=0$ and $\dot{y}<0$. Moreover, in order to see the consequences of such orbits on the dynamics from a global point of view, we will also consider the iterates obtained from orbits not related with ejection/collision orbits. More precisely, 
on the one hand, we take initial conditions along the $x$ axis (with $v=0$ and $u<0$ obtained from the fixed value of $h$ ). On the other hand, since for $h>h_{1}$, there exist the corresponding unstable Lyapunov periodic orbit (LPO1) and their stable and unstable invariant manifolds, we compute them all.

For example, for $K=0.1$ and according to the value of $h$ we will distinguish between different phenomena:

(i) We take $h=-1.4 \in\left(h_{1}, h_{2}\right)$. See Figure 11. On the top left plot we can see the PSP obtained from the ejection orbits in $W^{u}\left(S^{+}\right)$. We may conclude that the ejection orbits reach maximum values of $r$ ranging approximately in the interval $(0.68,0.72)$ and minimum values that range from 0 (ECO) to small values corresponding to close passages to the origin. On the top right plot, we show the PSP of the $W^{u}\left(S^{+}\right)$(in black) together with the stable and unstable manifolds of the LPO1 (in dark blue) as well as some region of confined invariant curves (in red), which are known to exist for small values of $K$ (see [1]), and the stable LPO2s and the surrounding invariant curves and islands (in light blue). We plot the single LPO2s in the bottom right plot. We also plot a zoom of the PSP in the bottom left plot. Actually, we see an outer region of red invariant curves -surrounding the $W^{u, s}\left(S^{+,-}\right)$- and an inner one. For this level of the energy $h$, we can conclude that all the to and from motion coming from the manifolds of $W^{u, s}\left(S^{+,-}\right)$will be confined since the outer red invariant curves (limiting the black ejection orbits) act as barriers. It is also clear that there is a rich structure of heteroclinic orbits and escape orbits just taking into account the manifolds of the LPO1. For smaller values of $h$, even the manifolds of the LPO1 are confined by a bigger region of invariant curves (see more details in $[1])$.

(ii) For bigger values of $h$, say $h=-0.8>h_{2}$, and concerning the set of ejection orbits, $W^{u}\left(S^{+}\right)$, we plot in Figure 12 (first row left) some iterates on the PSP. We can see that there exist some ejection orbits that remain bounded, in a typical rossette shape (see for example Figure 5 left for a different value 470 of $h$ ), escape orbits (see Figure 5 right) and of course ECO. In Figure 12 (first row right), we plot a higher number of iterates obtained from both $W^{u}\left(S^{+}\right)$ 
and $W^{s}\left(S_{-}\right)$. On the one hand, from this plot we infer a high richness of heteroclinic orbits (that is ECO belonging to both manifolds) that reach big values of $r$. For example we plot in Figure 12 (second row) an ECO in $(x, y)$ coordinates (left) and in $(\tau, r)$ variables (right) (the range in $\tau$ should be infinite of course but we just plot a finite one). On the other hand, if we also consider the global PSP (taking other initial conditions and the manifolds of the LPO1), we remark that the outer region of invariant curves has disappeared and that the mixing between the ejection orbits and the manifolds of the LPO1 is clearly visible (see Figure 12 third row). In particular, orbits are highly sensitive to small variations in initial conditions. For example, we plot in $(\tau, r)$ variables, a nearby orbit of the previous ECO, for a big range of time (see Figure 12 fourth row). We clearly see the erratic excursions far and close to the origin, and it finally seems to escape; actually there is no certainty that this orbit escapes, but if it reaches a high enough value of $r$, it is considered that it ionizes. So, for this level of the energy, both the ejection orbits and the manifolds of the LPO1 orbit are responsible not only for erratic to and fro motion but also for to and fro motion, for a range of time, plus ionization (or final escape). We also observe the LPO2s passes very close to the origin (see Figure 11) and becomes unstable, so the previous invariant curves around such orbit have disappeared (see the light blue point in Figure 12 third row).

(iii) Finally, for higher values of $h$, for example $h=-0.5$, most of the ejection orbits become unbounded and get completely mixed with the manifolds of the LPO1. See, in Figure 13 left. The inner red region of invariant curves still remains (see the red area in Figure 13) right, and, for this level of energy, the LPO2s is again stable. See the $(x, y)$ projection of the orbit in Figure 11 and the invariant curves around the associated fixed point in the PSP (light blue) in Figure 13 right.

As a final remark, we mention that the numerical simulations shown in this paper correspond to the particular case $K=0.1$. However, this is not any special value. The analysis of ejection/collision orbits for other values of $K$ would be essentially the same and the code works, of course, exactly the same. However, 
but for smaller values of $K$, the system is closer to integrable (when $K=0$ ) so it is harder to distinguish between the non-integrable typical behaviour and the integrable one (the orbits for $h<0$ become invariant curves, since they are keplerian ellipses in rotating coordinates). For bigger values of $K$, in particular for $K>\left(3^{-4 / 3}\right) / 2$, the equilibrium point $L_{2}$ (which was a center $\times$ center for smaller values of $K$ ) becomes a complex saddle. So the analysis of the global dynamics should take into account this feature, but this is the purpose of a future paper.

\section{Conclusions}

We have shown how the $n$-ECO may be regarded as the skeleton of to and fro motion. Just pairs of such orbits appear for any given $n$ and small values of the energy $h$, but intricated families and bifurcations show up for higher values

\section{Acknowledgments}

This work was supported by the Spanish MINECO/FEDER grant MTM201565715-P and the Catalan grant 2014SGR-00504.

\section{References}

[1] Barrabés, E., Ollé, M., Borondo, F., Farrelly, D., Mondelo, J. M. Phase space structure of the hydrogen atom in a circularly polarized microwave field. Phys D 2012;241:333-349.

[2] Brunello, A. F., Uzer, T., Farrelly, D. Hydrogen atom in circularly polarized microwaves: Chaotic ionization via core scattering. Phys Rev A 1997;55(5):3730-3745. 
[3] Bruno, A. D. The Restricted 3-Body problem. New York: Walter de Gruyter; 1994.

[4] Cole, D. C., Zou, Y. Perturbation analysis and simulation study of the effects of phase on the classical hydrogen atom interacting with circularly polarized electromagnetic radiation. J. Sci. Comput.;2004:145-172

[5] Hénon, M. Generating families in the Restricted Three-Body problem. Berlin: Lecture Notes in Physics, Springer; 1997.

[6] Hénon, M. Generating families in the Restricted Three-Body problem. II. Quantitative study of bifurcations. Berlin: Lecture Notes in Physics, Springer; 2001.

[7] Jorba, A. and Zou, M. A sofware package for the numerical integratio of ODEs by means of high-order Taylor methods. Experiment. Maths. 2005;14:99-117.

[8] Lacomba, E. A. and Llibre, J. Transversal Ejection-Collision Orbits for the Restricted Problem and the Hill's Problem with Applications. J Differ Equations 1988;74:69-85.

[9] Llibre, J. On the Restricted Three-Body Problem when the Mass Parameter is Small. Celestial Mech Dynam Astronom 1982;28:83-105.

[10] Llibre, J, and Martinez-Alfaro, J. Ejection and collision orbits of the spatial RTBP. Celestial Mech 1985;35:113-128.

[11] Llibre, J. and Pinyol, C. On the Elliptic Restricted Three-Body Problem. Celestial Mech Dynam Astronom 1990;48:319-345.

[12] Mauger, F., Chandre, C., Uzer, T. Recollisions and correlated double ionization with circularly polarized light. Phys. Rev. Lett. 2010;105:083002.

[13] Meyer, K, R., Hall, G. R. Introduction to Hamiltonian dynamical systems and the $N$-body problem. Springer-Verlag, New York; 1992. 
[14] Ollé, M., Rodriguez, O., Soler, J. Ejection-collision orbits in the RTBP. Submitted. 2017.

[15] Pinyol, C. Ejection-collision orbits with the more massive primary in the planar elliptic restricted three-body problem. Celestial Mech Dynam. Astronom 1995;61:315-331.

[16] Szebehely, V. Theory of orbits. Academy Press, Inc., New York; 1967. 